\title{
The correlation of respiratory system compliance and mortality in COVID-19 acute respiratory distress syndrome: do phenotypes really exist?
}

\begin{abstract}
Background: Recent literature suggests respiratory system compliance (Crs) based phenotypes exist among COVID-19 ARDS patients. We sought to determine whether these phenotypes exist and whether Crs predicts mortality.

Methods: A retrospective observational cohort study of 111 COVID-19 ARDS patients admitted March 11-July 8, 2020. Crs was averaged for the first 72-hours of mechanical ventilation. $\mathrm{Crs}<30 \mathrm{ml} / \mathrm{cmH}_{2} \mathrm{O}$ was defined as poor $\mathrm{Crs}$ (phenotype- $\mathrm{H}$ ) whereas $\mathrm{Crs} \geq 30 \mathrm{ml} /$ $\mathrm{cmH}_{2} \mathrm{O}$ as preserved $\mathrm{Crs}$ (phenotype-L).
\end{abstract}

Results: 111 COVID-19 ARDS patients were included, 40 phenotype-H and 71 phenotype- $\mathrm{L}$. Both the mean $\mathrm{Pa}_{\mathrm{O} 2} / \mathrm{Fi}_{\mathrm{O} 2}$ ratio for the first 72-hours of mechanical ventilation and the $\mathrm{Pa} / \mathrm{Fi}_{\mathrm{O} 2}$ ratio hospital nadir were lower in phenotype-H than L(115[IQR87] vs 165 [87], $p=0.016),(63$ [32] vs 75 [59], $p=0.026)$. There were no difference in characteristics, diagnostic studies, or complications between groups. Twenty-seven (67.5\%) phenotype-H patients died vs 37(52.1\%) phenotype- $\mathrm{L}(p=0.115)$. Multivariable regression did not reveal a mortality difference between phenotypes; however, a 2-fold mortality increase was noted in $\mathrm{Crs}<20$ vs $>50 \mathrm{ml} / \mathrm{cmH}_{2} \mathrm{O}$ when analyzing ordinal Crs groups. Moving up one group level (ex. Crs30-39.9ml/ $\mathrm{cmH}_{2} \mathrm{O}$ to $40-49.9 \mathrm{ml} / \mathrm{cmH}_{2} \mathrm{O}$ ), was marginally associated with $14 \%$ lower risk of death $(\mathrm{RR}=0.86,95 \% \mathrm{CI} 0.72,1.01, p=0.065)$. This attenuated $(\mathrm{RR}=0.94$, $95 \% \mathrm{CI} 0.80,1.11)$ when adjusting for $\mathrm{pH}$ nadir and $\mathrm{Pa}_{\mathrm{O} 2} / \mathrm{Fi}_{\mathrm{O} 2}$ ratio nadir

Conclusion: We identified a spectrum of Crs in COVID-19 ARDS similar to Crs distribution in non-COVID-19 ARDS. While we identified increasing mortality as Crs decreased, there was no specific threshold marking significantly different mortality based on phenotype. We therefore would not define COVID-19 ARDS patients by phenotypes-H or L and would not stray from traditional ARDS ventilator management strategies.

Keywords: COVID-19, corona virus, acute respiratory distress syndrome, respiratory system compliance, pulmonary compliance, mortality, intensive care unit, critical care, mechanical ventilation
Volume 8 Issue 2 - 202 I

\author{
Rachel L. Choron MD,' Stephen A. lacono \\ MD, ' Alexander Cong, ${ }^{2}$ Christopher G. \\ Bargoud MD,' Amanda L. Teichman MD,' \\ Nicole J. Krumrei MD FACS,' Michelle T. \\ Bover Manderski PhD MPH, ${ }^{3}$ Michael B. \\ Rodricks MD FNCS,' Rajan Gupta MD FACS \\ MHCDS,' Matthew E. Lissauer MD FACS \\ FCCM'
}

'Division of Acute Care Surgery, Department of Surgery, Rutgers Robert Wood Johnson Medical School, New Brunswick, New Jersey, USA

${ }^{2}$ Rutgers Robert Wood Johnson Medical School, New Brunswick, New Jersey, USA

${ }^{3}$ Department of Biostatistics and Epidemiology, Rutgers School of Public Health, New Brunswick, New Jersey, USA

Correspondence: Rachel L. Choron, MD,Assistant Professor, Department of Surgery, Rutgers Robert Wood Johnson Medical School, 125 Patterson Street, Suite 6300, New Brunswick, NJ 0890।, Tel 9|4-584-8552, Fax 732-235-2964, Email Rachel.Choron@gmail.com

Received: May 05, 202I | Published: May 20, 202 I

\section{Introduction}

The coronavirus (COVID-19) pandemic was first reported in Wuhan, China in December 2019 and rapidly spread worldwide. ${ }^{1,2}$ As of April 21st, 2021, there have been over 144 million cases globally, including 31 million cases and 569,000 deaths in the United States. ${ }^{3}$ Mortality in intensive care unit (ICU) patients with COVID-19 remains high despite advances in treatment strategies. Mortality ranges from $30-60 \%$ among COVID-19 ICU patients and is even higher in patients requiring mechanical ventilation.-9

A large percentage of COVID-19 patients who develop respiratory failure and hypoxemia have been diagnosed with acute respiratory distress syndrome (ARDS) and treated accordingly; however, few data exist correlating respiratory pathophysiology to clinical features and ventilator mechanics in COVID-19. Patients presenting with non-COVID-19 ARDS typically have low pulmonary compliance and loss of aerated tissue available for ventilation. ${ }^{10,11}$ While most patients with COVID-19 ARDS present similarly to non-COVID-19 ARDS, as defined by the Berlin Criteria, ${ }^{12}$ it has been postulated that some COVID-19 patients do not fit the classic ARDS phenotype characterized by poor pulmonary compliance. Several studies have demonstrated that some hypoxemic COVID-19 patients with respiratory failure requiring mechanical ventilation have higher than expected pulmonary compliance when compared to non-COVID-19 ARDS patients. ${ }^{13-15}$

Based on these observations, Gattinoni et al. proposed two distinct phenotypes among patients with COVID-19 pneumonia who met the Berlin criteria for ARDS. ${ }^{16}$ The proposed "L phenotype" was defined as low elastance and high compliance; conversely the "H phenotype" was defined as high elastance and low compliance. ${ }^{16}$ It was further hypothesized that unlike $\mathrm{H}$ phenotype patients who benefit from classic ARDS lung protective ventilator strategies, L phenotype patients would paradoxically benefit from low PEEP and high tidal volume to compensate for increased dead space caused by hypoxic pulmonary vasoconstriction, which is contrary to traditional ARDS management.

Our primary objective was to determine whether respiratory system compliance (Crs) based phenotypes still exist among COVID-19 ICU patients during the first 72-hour period after the initiation of mechanical ventilation in our patient cohort. Our secondary objective was to determine whether Crs was related to mortality. 


\section{Methods}

\section{Patient population and setting}

This was a retrospective observational study of the first 111 consecutive COVID-19 patients admitted to the ICU who required mechanical ventilation. Patients were admitted from March 11, 2020 to July 8,2020 . The final study follow-up date was November $23^{\text {rd }}$, 2020. The Institutional Review Board approved this study. Patients who met inclusion criteria were 18 years of age or older, admitted to the ICU, SARS-CoV-19 positive confirmed by polymerase chain reaction (PCR) testing of nasopharyngeal swab, mechanically ventilated for respiratory failure, diagnosed with ARDS as per the Berlin definition, ${ }^{12}$ and discharged from the ICU or died at the final date of follow-up. The exclusion criterion was COVID-19 ICU patients who did not require mechanical ventilation. This study was performed in a 297-bed community hospital in Central New Jersey that is affiliated with a large academic tertiary care center. During the height of the pandemic, the ICU was expanded to three times its typical capacity during mid-April.

\section{Data collection and definitions}

Patient information was collected from the electronic medical record (Allscripts-Sunrise Clinical Manager, Chicago, IL). The data collected included patient demographics, past medical history, vital signs, laboratory testing, therapies utilized for treatment of COVID-19, ICU length of stay, hospital length of stay, days to initiation of mechanical ventilation, days of mechanical ventilation, mortality, and disposition. Complications collected included acute respiratory distress syndrome (ARDS) defined by the Berlin criteria, ${ }^{12}$ acute kidney injury as defined by the Kidney Disease: Improving Global Outcomes (KDIGO) definition, ${ }^{17}$ culture proven infection, image proven venous thromboembolism, hemorrhagic events, and need for tracheostomy.

Ventilator data, arterial blood gases, and respiratory mechanics were collected during the first 72 hours following intubation for each patient. The $\mathrm{Crs}\left(\mathrm{ml} / \mathrm{cm} \mathrm{H}_{2} \mathrm{O}\right)$ was defined as the tidal volume $\left(\mathrm{V}_{\mathrm{T}}\right)$ divided by the difference between plateau pressure and positive end expiratory pressure (PEEP). Average Crs was obtained for each patient over the first 72 hours they were mechanically ventilated. $\mathrm{Crs}<30 \mathrm{ml} /$

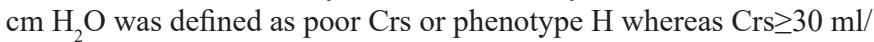
$\mathrm{cm} \mathrm{H}_{2} \mathrm{O}$ was defined as preserved $\mathrm{Crs}$ or phenotype $\mathrm{L}$.

All critically ill COVID-19 patients were cared for by a multidisciplinary team led by a board certified intensivist 24 hours a day seven days a week. Clinical practice patterns involved protective lung ventilation strategies as recommended by the ARDS network, however as this was a retrospective observational study without intervention, practice patterns were left to the discretion of the attending intensivist.

\section{Statistical analysis}

The distributions of baseline characteristics, vital signs, laboratory results, treatments, and outcomes were calculated for the entire study population, for phenotype $\mathrm{H}$ patients with $\mathrm{Crs}<30 \mathrm{ml} / \mathrm{cm} \mathrm{H}_{2} \mathrm{O}$, and for phenotype L patients with $\mathrm{Crs} \geq 30 \mathrm{ml} / \mathrm{cm} \mathrm{H}_{2} \mathrm{O}$. Frequency and percentages are reported for categorical variables, and median and interquartile range (IQR) are reported for continuous variables, since nearly all were determined to be nonnormally distributed according to the Shapiro-Wilk statistic. Bivariate comparisons of categorical and continuous variables were tested using Pearson's chi-square statistic (or Fisher's exact test when warranted by small cell counts) or twosided Wilcoxon Rank-Sum statistics, respectively.

Mortality along with other continuous variable outcomes (hospital days prior to mechanical ventilation, mechanical ventilator days, ICU length of stay, and hospital length of stay) were compared for phenotypes $\mathrm{H}$ and $\mathrm{L}$. To determine whether a Crs threshold other than $30 \mathrm{ml} / \mathrm{cm} \mathrm{H}_{2} \mathrm{O}$ impacted mortality and outcomes, patients were divided into five ordinal groups (i.e., $<20,20$ to $<30,30$ to $<40,40$ to $<50$, and $\geq 50 \mathrm{ml} / \mathrm{cm} \mathrm{H}_{2} \mathrm{O}$ ) based on Crs and Kruskal-Wallis test was used to assess bivariate association with continuous variable outcomes. Adjusted relative risks were computed using multivariable modified (robust variance estimator) Poisson regression models; first analysis was with $\mathrm{Crs} \geq 30 \mathrm{ml} / \mathrm{cm} \mathrm{H}_{2} \mathrm{O}$ vs $<30 \mathrm{ml} / \mathrm{cm} \mathrm{H}_{2} \mathrm{O}$ (Model 1), adjusted for $\mathrm{pH}$ nadir $<7.2$ and lowest $\mathrm{Pa}_{\mathrm{O} 2} / \mathrm{Fi}_{\mathrm{O} 2}$ Ratio (Model 2), the second analysis evaluated the 5-level ordinal Crs groups. All analyses were performed using SAS software version 9.4 (SAS Institute, Cary, NC).

\section{Results}

At the final date of follow-up, 111 COVID-19 positive ICU patients met study inclusion criteria which included requiring mechanical ventilation and meeting the Berlin criteria for ARDS. The median age for the overall population was 64 years (IQR 17) and the majority were male $(76,68.5 \%)$. Most patients were Caucasian (50, $45.1 \%)$, followed by Hispanic $(39,35.1 \%)$, and Black $(15,13.5 \%)$ (Table 1A). Of the total population, 40 patients $(36 \%)$ were classified as phenotype $\mathrm{H}\left(\mathrm{Crs}<30 \mathrm{ml} / \mathrm{cm} \mathrm{H}_{2} \mathrm{O}\right)$ and $71(64 \%)$ were classified as phenotype $\mathrm{L}\left(\mathrm{Crs} \geq 30 \mathrm{ml} / \mathrm{cm} \mathrm{H}_{2} \mathrm{O}\right)$.

Table Ia Characteristics of mechanically ventilated ARDS patients with COVID-19 stratified by Crs

\begin{tabular}{|c|c|c|c|c|}
\hline & $\begin{array}{l}\text { All COVID-19 } \\
\text { ARDS Patients } \\
(\mathrm{n}=|1| \mathrm{I})\end{array}$ & $\begin{array}{l}\text { Patients with Crs } \\
<30 \mathrm{ml} / \mathrm{cm} \mathrm{H}_{2} \mathrm{O} \\
\text { Phenotype } \mathrm{H}_{(\mathrm{n}=40)}\end{array}$ & $\begin{array}{l}\text { Patients with Crs } \\
\geq 30 \mathrm{ml} / \mathrm{cm} \mathrm{H}_{2} \mathrm{O} \\
\text { Phenotype } \mathrm{L}(\mathrm{n}=7 \mathrm{I})\end{array}$ & p-value \\
\hline Age, median (IQR), years & $64(17)$ & $66(14.5)$ & $61(16)$ & 0.092 \\
\hline Sex, male (n, \%) & $76(68.5)$ & $22(55)$ & $54(76.1)$ & 0.022 \\
\hline \multicolumn{5}{|l|}{ Race/Ethnicity } \\
\hline Caucasian & $50(45.1)$ & $20(50)$ & $30(42.3)$ & 0.645 \\
\hline Black & $15(13.5)$ & $6(15)$ & $9(12.7)$ & \\
\hline Hispanic & $39(35.1)$ & II (27.5) & $28(39.4)$ & \\
\hline Asian & $7(6.3)$ & $3(7.5)$ & $4(5.6)$ & \\
\hline Body Mass Index, median (IQR), kg/m² & $30.8(10.3)$ & $29.4(9.5)$ & $30.8(10.1)$ & 0.338 \\
\hline
\end{tabular}


Table Continued...

\begin{tabular}{|c|c|c|c|c|}
\hline & $\begin{array}{l}\text { All COVID-19 } \\
\text { ARDS Patients } \\
(n=111)\end{array}$ & $\begin{array}{l}\text { Patients with Crs } \\
<30 \mathrm{ml} / \mathrm{cm} \mathrm{H}_{2} \mathrm{O} \\
\text { Phenotype } \mathrm{H}_{(\mathrm{n}=40)}\end{array}$ & $\begin{array}{l}\text { Patients with Crs } \\
\geq 30 \mathrm{ml} / \mathrm{cm} \mathrm{H}_{2} \mathrm{O} \\
\text { Phenotype } \mathrm{L}(\mathrm{n}=7 \mathrm{I})\end{array}$ & p-value \\
\hline \multicolumn{5}{|l|}{ Comorbities, (n, \%) } \\
\hline Diabetes & $49(44.1)$ & $20(50)$ & $29(40.9)$ & 0.351 \\
\hline Hypertension & $68(6 \mid .3)$ & $28(70)$ & $40(56.3)$ & 0.156 \\
\hline COPD/Asthma & II (9.9) & $4(10)$ & $7(9.9)$ & 1.000 \\
\hline $\begin{array}{l}\text { Cardiovascular Disease (CAD, MI, } \\
\mathrm{HF})\end{array}$ & $26(23.4)$ & II (27.5) & $15(21.1)$ & 0.449 \\
\hline Smoking History & $22(19.8)$ & $7(17.5)$ & $15(21.1)$ & 0.645 \\
\hline Chronic Kidney Disease & $14(12.6)$ & $3(7.5)$ & II (I5.5) & 0.223 \\
\hline
\end{tabular}

The most common comorbidities among all COVID-19 ICU patients were hypertension $(61.3 \%)$, diabetes $(44.1 \%)$, and obesity with a median body mass index $30.8 \mathrm{~kg} / \mathrm{m}^{2}$ (IQR 10.3). Baseline characteristics, listed in Table 1A, were similar among phenotypes $\mathrm{H}$ and L. Admission vital signs and laboratory values were also similar between the two groups (Table 1B). While not statistically significant, phenotype $\mathrm{H}$ had a lower heart rate compared to phenotype $\mathrm{L}(94.5$ beats per minute [IQR25] vs 101 [29], $p=0.059$ ) and phenotype $\mathrm{H}$ trended toward a lower mean arterial pressure $(89 \mathrm{mmHg}$ [26] vs 95 [21], $p=0.057)$.

Table I b Vital Signs, Laboratory Results, and Gas Exchange Data Stratified by Crs

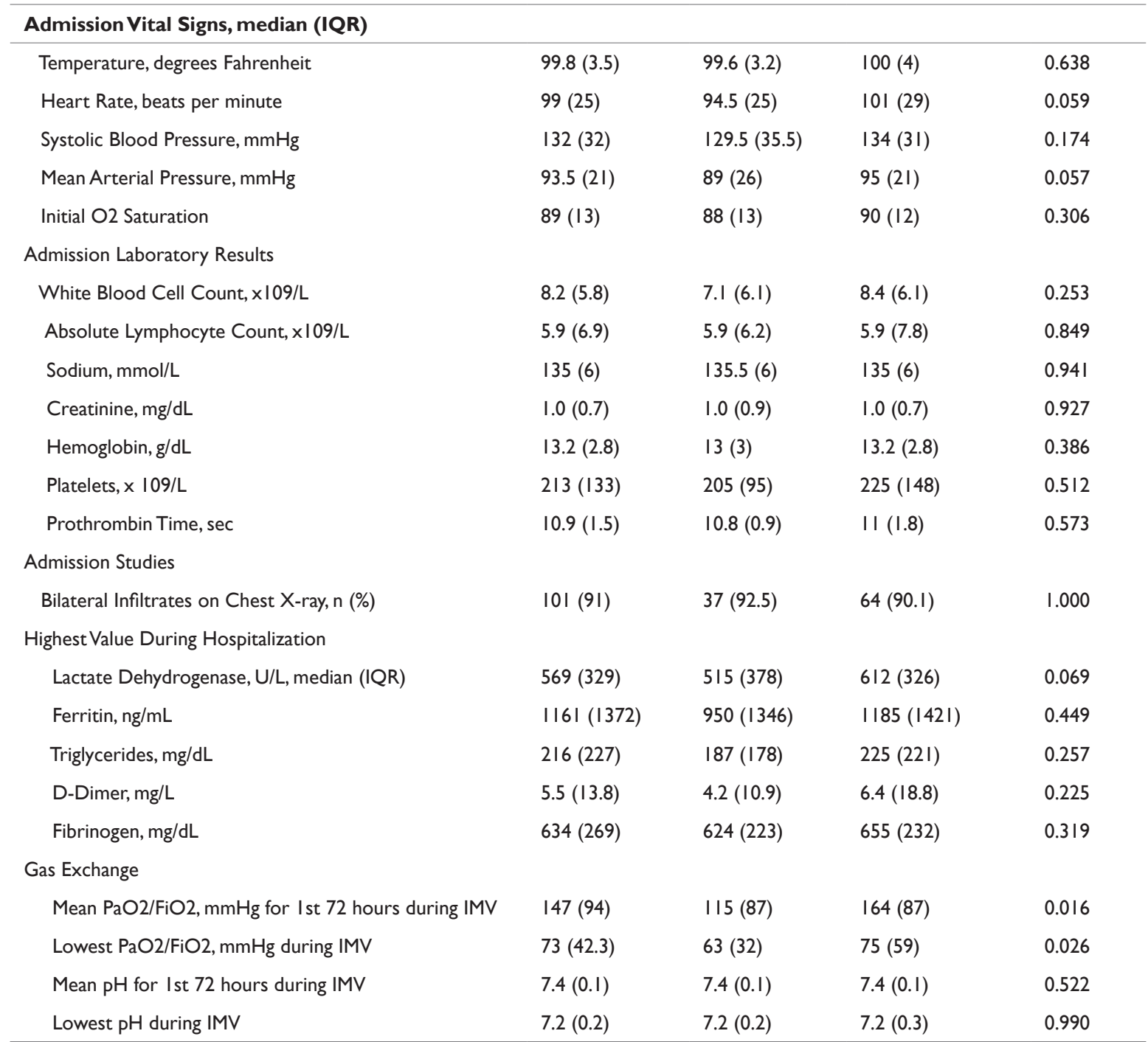

Abbreviations: ARDS, acute respiratory distress syndrome; Crs, respiratory system compliance; IQR, interquartile range; CAD, coronary artery disease; MI, myocardial infarction; HF, heart failure; IMV, invasive mechanical ventilation 
Both phenotype $\mathrm{H}$ and $\mathrm{L}$ patients were critically ill. The majority of patients had shock requiring vasopressors during ICU admission (88.9\%). All patients had ARDS; 83 (74.8\%) had severe ARDS based on Berlin criteria. The mean $\mathrm{Pa}_{\mathrm{O} 2} / \mathrm{Fi}_{\mathrm{O} 2}$ ratio for the first 72 hours of mechanical ventilation was significantly lower in phenotype $\mathrm{H}$ (115 [87] vs 165 [87], $p=0.016$ ). Likewise, the $\mathrm{Pa}_{\mathrm{O} 2} / \mathrm{Fi}_{\mathrm{O} 2}$ ratio nadir for the entire mechanical ventilation course was lower in phenotype $\mathrm{H}$ than $\mathrm{L}$ (63 [32] vs 75 [59], $p=0.026)$.
Complications were similar between both phenotype $\mathrm{H}$ and $\mathrm{L}$ groups (Table 2). Fifty-one (46.4\%) patients had concomitant bacterial pneumonia and 26 (23.4\%) had bacteremia. Nearly half (52, 47.3\%) had acute kidney injury and $32(28.8 \%)$ required renal replacement therapy. There was no difference among infectious, cardiac, or other complications between the two groups. While not statistically significant, fewer phenotype $\mathrm{H}$ patients underwent tracheostomy procedures than phenotype L (2 [5\%] vs 12 [16.9\%], $p=0.070)$.

Table 2 Complications of mechanically ventilated ARDS patients with COVID- 19 stratified by Crs

\begin{tabular}{|c|c|c|c|c|}
\hline & $\begin{array}{l}\text { All COVID-19 } \\
\text { ARDS Patients } \\
(\mathrm{n}=|| I)\end{array}$ & $\begin{array}{l}\text { Patients with Crs } \\
<30 \mathrm{ml} / \mathrm{cm} \mathrm{H}_{2} \mathrm{O} \\
\text { Phenotype } \mathrm{H}^{\prime}(\mathrm{n}=40)\end{array}$ & $\begin{array}{l}\text { Patients with Crs } \\
\geq 30 \mathrm{ml} / \mathrm{cm} \mathrm{H}_{2} \mathrm{O} \\
\text { Phenotype } L(n=7 \mathrm{l})\end{array}$ & p-value \\
\hline & n (\%) & $\mathrm{n}(\%)$ & n (\%) & \\
\hline \multicolumn{5}{|l|}{ ARDS } \\
\hline Mild ARDS & $5(4.5)$ & I (2.5) & $4(5.6)$ & 0.416 \\
\hline Moderate ARDS & $23(20.7)$ & $6(15)$ & $17(23.9)$ & 0.264 \\
\hline Severe ARDS & $83(74.8)$ & $33(82.5)$ & $50(70.4)$ & 0.160 \\
\hline Vasopressor Requirement & $97(88.9)$ & $36(92.3)$ & $61(87.1)$ & 0.532 \\
\hline \multicolumn{5}{|l|}{ Infectious Complications } \\
\hline Bacterial Pneumonia & $51(46.4)$ & I5 (37.5) & $36(51.4)$ & 0.159 \\
\hline Urinary Tract Infection & $24(21.6)$ & $10(25)$ & $14(19.7)$ & 0.516 \\
\hline Bacteremia & $26(23.4)$ & $10(25)$ & $16(22.5)$ & 0.769 \\
\hline Influenza & $2(1.8)$ & 0 & $2(2.8)$ & 0.535 \\
\hline Clostridium Difficile & $2(1.8)$ & 0 & $2(2.8)$ & 0.535 \\
\hline Acute Kidney Injury & $52(47.3)$ & $19(48.7)$ & $33(46.5)$ & 0.822 \\
\hline Kidney Replacement Therapy & $32(28.8)$ & $10(25)$ & $22(3 I)$ & 0.504 \\
\hline Acute Hepatic Injury & $9(8.1)$ & $5(12.5)$ & $4(5.6)$ & 0.279 \\
\hline \multicolumn{5}{|l|}{ Cardiac Complications } \\
\hline Arrhythmia & $34(30.6)$ & $8(20)$ & $26(36.6)$ & 0.068 \\
\hline Myocardial Infarction & $4(3.6)$ & I (2.5) & $3(4.2)$ & 1.000 \\
\hline Cardiomyopathy & $8(7.2)$ & $2(5)$ & $6(8.5)$ & 0.709 \\
\hline Pneumothorax & II (9.9) & $3(7.5)$ & $8(11.3)$ & 0.743 \\
\hline Seizures & $2(1.8)$ & I (2.5) & I (I.4) & 1.000 \\
\hline Deep Vein Thrombosis & $3(2.7)$ & 0 & $3(4.2)$ & 0.552 \\
\hline Pulmonary Embolism & $2(1.8)$ & 0 & $2(2.9)$ & 0.531 \\
\hline Tracheostomy & $14(12.6)$ & $2(5)$ & $12(16.9)$ & 0.070 \\
\hline
\end{tabular}

Abbreviations: ARDS, acute respiratory distress syndrome; Crs, respiratory system compliance; IQR, interquartile range

\section{Outcomes and mortality as related to respiratory system compliance}

The overall mortality was $57.7 \%$ for COVID-19 ARDS patients, with median mechanical ventilator days 10 [IQR11], ICU length of stay 12 days [12], and hospital length of stay 11 days [IQR 15] (Table
3A). There was no difference in ventilator days, ICU length of stay, hospital length of stay, or mortality when comparing phenotypes $\mathrm{H}$ and $\mathrm{L}$. Twenty-seven (67.5\%) phenotype $\mathrm{H}$ patients died as compared to $37(52.1 \%)$ phenotype L patients $(p=0.115)$. While there was no difference in mortality when stratifying respiratory system compliance based on phenotypes $\mathrm{H}$ and $\mathrm{L}$, there was a trend toward 
increasing mortality with decreasing respiratory system compliance when dividing the patients into ordinal groups (Table 3B, Figure 1). There was $40 \%$ mortality among patients with $\mathrm{Crs}>50 \mathrm{ml} / \mathrm{cm} \mathrm{H}_{2} \mathrm{O}$ vs
$55.1 \%$ mortality with $\mathrm{Crs} 30-39.9 \mathrm{ml} / \mathrm{cm} \mathrm{H}_{2} \mathrm{O}$ and $80 \%$ mortality with $\mathrm{Crs}<20 \mathrm{ml} / \mathrm{cm} \mathrm{H}_{2} \mathrm{O}$.

Table 3A Outcomes of mechanically ventilated ARDS patients with COVID-19 stratified by Crs

\begin{tabular}{|c|c|c|c|c|}
\hline & $\begin{array}{l}\text { All COVID-19 } \\
\text { ARDS Patients } \\
(n=111)\end{array}$ & $\begin{array}{l}\text { Patients with Crs }<30 \\
\mathrm{ml} / \mathrm{cm} \mathrm{H}_{2} \mathrm{O} \text { Phenotype } \\
\mathrm{H}(\mathrm{n}=40)\end{array}$ & $\begin{array}{l}\text { Patients with Crs } \\
\geq 30 \mathrm{ml} / \mathrm{cm} \mathrm{H}_{2} \mathrm{O} \\
\text { Phenotype } L(n=7 \mathrm{I})\end{array}$ & p-value \\
\hline Hospital Days Prior to IMV, median (IQR) & $2.0(3.0)$ & $1.0(3.0)$ & $2.0(4.0)$ & 0.823 \\
\hline Mechanical Ventilator Days & $10(11)$ & $11.5(9)$ & $9(13)$ & 0.880 \\
\hline Intensive Care Unit Length of Stay, days & $12(12)$ & $13.5(10)$ & $12(17)$ & 0.958 \\
\hline Hospital Length of Stay, days & $17(15)$ & $16(15)$ & $17(16)$ & 0.305 \\
\hline Mortality, n (\%) & $64(57.7)$ & $27(67.5)$ & $37(52.1)$ & 0.115 \\
\hline Mortality among proned patients $(n=3 \mathrm{I})$ & $22 / 3 I(7 I)$ & $9 / 11(81.8)$ & $13 / 20(65)$ & 0.429 \\
\hline
\end{tabular}

Table 3B Outcomes of Mechanically Ventilated ARDS Patients with COVID-19 Based on Crs Group

\begin{tabular}{|c|c|c|c|c|c|c|}
\hline & $\begin{array}{l}\text { Crs }<20 \mathrm{ml} / \\
\mathrm{cm} \mathrm{H} \mathrm{H}_{2} \mathrm{O}(\mathrm{n}=5)\end{array}$ & 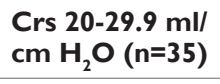 & $\begin{array}{l}\text { Crs 30-39.9 ml/ } \\
\mathrm{cm} \mathrm{H}_{2} \mathrm{O}(\mathrm{n}=49)\end{array}$ & $\begin{array}{l}\text { Crs } 40-49.9 \mathrm{ml} / \\
\mathrm{cm} \mathrm{H}_{2} \mathrm{O}(\mathrm{n}=7)\end{array}$ & $\begin{array}{l}\mathrm{Crs}>50 \mathrm{ml} / \mathrm{cm} \\
\mathrm{H}_{2} \mathrm{O}(\mathrm{n}=15)\end{array}$ & p-value \\
\hline $\begin{array}{l}\text { Hospital Days Prior to IMV, } \\
\text { median (IQR) }\end{array}$ & $2(2)$ & I (3) & I (4) & $4(5)$ & $2(3)$ & 0.777 \\
\hline Mechanical Ventilator Days & $7(3)$ & $12(9)$ & $10(12)$ & $8(19)$ & $6(16)$ & 0.681 \\
\hline $\begin{array}{l}\text { Intensive Care Unit Length of } \\
\text { Stay, days }\end{array}$ & $8(4)$ & $14(10)$ & $12(14)$ & $8(19)$ & $8(21)$ & 0.707 \\
\hline Hospital Length of Stay, days & II (17) & $16(14)$ & $17(15)$ & $15(15)$ & $18(30)$ & 0.735 \\
\hline Mortality, n (\%) & $4(80)$ & $23(65.7)$ & $27(55.1)$ & $4(57.1)$ & $6(40)$ & 0.681 \\
\hline $\begin{array}{l}\text { Mortality among proned } \\
\text { patients }\end{array}$ & $\mathrm{I} / \mathrm{I}(100)$ & $8 / 10(80)$ & II/I5 (73.3) & 0 & $2 / 5(40)$ & \\
\hline
\end{tabular}

Abbreviations: ARDS, acute respiratory distress syndrome; Crs, respiratory system compliance; IQR, interquartile range; IMV, invasive mechanical ventilation

\section{Increasing Mortality with Decreasing Pulmonary Compliance in Mechanically Ventilated COVID-19 Patients}

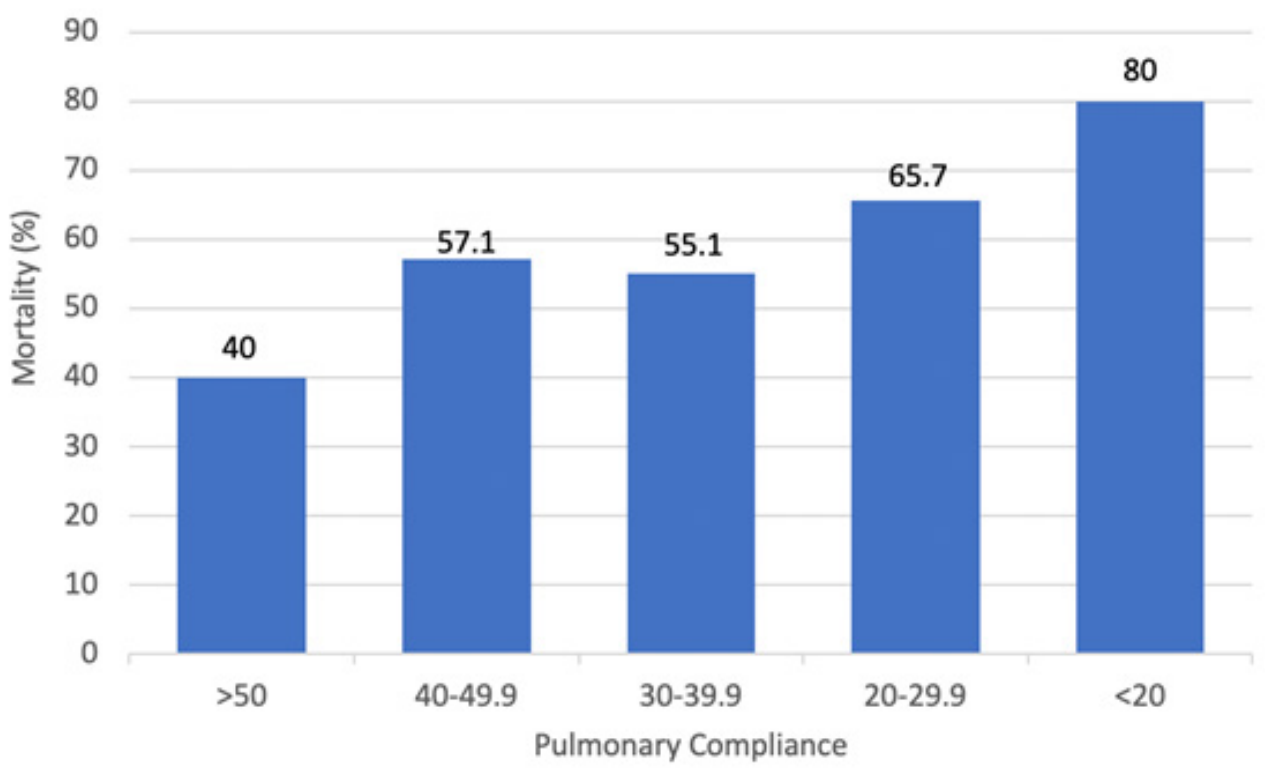

Figure I Increasing mortality demonstrated among mechanically ventilated COVID-19 ARDS patients with decreasing respiratory system compliance. 


\section{Multivariable analysis}

In multivariable analysis models one and two, respiratory system compliance was defined as phenotype $\mathrm{H}$ and $\mathrm{L}$. Using these models Crs was not associated with mortality and neither was $\mathrm{Pa}_{\mathrm{O} 2} / \mathrm{Fi}_{\mathrm{O} 2}$ ratio nadir; whereas abnormal $\mathrm{pH}$ was associated with a 2-fold increased risk for mortality (Table 4A). When evaluating Crs ordinally instead of as binary phenotype, a 2-fold increase in mortality was noted in
Crs $<20$ vs $>50 \mathrm{ml} / \mathrm{cm} \mathrm{H}_{2} \mathrm{O}$ (RR 2.00, 95\% CI 0.94-4.27); however, this association did not hold upon adjusting for $\mathrm{pH}$ nadir and $\mathrm{Pa}_{\mathrm{O} 2} /$ $\mathrm{Fi}_{\mathrm{O} 2}$ ratio nadir (Table 4B). Ordinally, each additional group level (ex. Crs $30-39.9 \mathrm{ml} / \mathrm{cm} \mathrm{H}_{2} \mathrm{O}$ to $40-49.9 \mathrm{ml} / \mathrm{cm} \mathrm{H}_{2} \mathrm{O}$ ) was marginally associated with a $14 \%$ lower risk of death $(\mathrm{RR}=0.86,95 \% \mathrm{CI} 0.72$, $1.01, p=0.065)$, this attenuates $(\mathrm{RR}=0.94,95 \% \mathrm{CI} 0.80,1.11)$ when adjusting for $\mathrm{pH}$ nadir and $\mathrm{Pa}_{\mathrm{O} 2} / \mathrm{Fi}_{\mathrm{O} 2}$ ratio nadir.

Table 4a Multivariable modified poisson regression analysis of mechanically ventilated ARDS patients with COVID-I9

\begin{tabular}{lllll}
\hline & Model I & \multicolumn{3}{c}{ Model 2 } \\
\hline & RR & $95 \% \mathrm{Cl}$ & RR & $95 \% \mathrm{Cl}$ \\
Compliance, $\mathrm{Crs}>30 \mathrm{vs}<30 \mathrm{ml} / \mathrm{cmH}_{2} \mathrm{O}$ & 0.77 & $0.57,1.05$ & 0.90 & $0.68,1.19$ \\
$\mathrm{PH} \mathrm{Nadir}<7.2$ & & & 2.04 & $1.27,3.28$ \\
Lowest $\mathrm{Pa}_{\mathrm{O} 2} / \mathrm{Fi}_{\mathrm{O} 2}$ Ratio & & & 0.99 & $0.99,1.00$
\end{tabular}

Table 4b Multivariable modified poisson regression analysis of mechanically ventilated ARDS patients with COVID-I 9 based on ordinal group

\begin{tabular}{|c|c|c|c|c|}
\hline & Model 3 & & Mod & \\
\hline & $\mathrm{RR}$ & $95 \% \mathrm{Cl}$ & $\mathrm{RR}$ & $95 \% \mathrm{Cl}$ \\
\hline \multicolumn{5}{|l|}{ Compliance } \\
\hline $\mathrm{Crs}<20 \mathrm{ml} / \mathrm{cmH}_{2} \mathrm{O}$ & 2.00 & $0.94,4.27$ & 1.16 & $0.50,2.17$ \\
\hline Crs $20-29.9 \mathrm{ml} / \mathrm{cmH}_{2} \mathrm{O}$ & 1.64 & $0.85,3.19$ & I.31 & $0.67,2.54$ \\
\hline Crs $30-39.9 \mathrm{ml} / \mathrm{cmH}_{2} \mathrm{O}$ & 1.38 & $0.71,2.69$ & 1.19 & $0.62,2.29$ \\
\hline Crs $40-49.9 \mathrm{ml} / \mathrm{cmH}_{2} \mathrm{O}$ & 1.43 & $0.59,3.49$ & 1.22 & $0.51,2.89$ \\
\hline $\mathrm{Crs}>50 \mathrm{ml} / \mathrm{cmH}_{2} \mathrm{O}$ & reference & & & \\
\hline $\mathrm{pH}$ Nadir $<7.2$ & & & 2.05 & $1.27,3.33$ \\
\hline Lowest $\mathrm{Pa}_{\mathrm{O} 2} / \mathrm{Fi}_{\mathrm{O} 2}$ Ratio & & & 0.99 & $0.99,1.00$ \\
\hline
\end{tabular}

*Ordinal trend: Model 3 RR 0.86 (95\% Cl 0.72-I.0I, p=0.065); Model 4 RR 0.94 (95\% Cl 0.80-I.II, p=0.458)

Abbreviations: ARDS, acute respiratory distress syndrome; $\mathrm{Crs}$, respiratory system compliance; $\mathrm{RR}$, risk ratio; $\mathrm{Cl}$, confidence interval

\section{Discussion}

While some argue that the mechanical properties of COVID-19 affected lungs are unique, recent studies have shown that patients with COVID-19 ARDS versus non-COVID-19 ARDS are similar. ${ }^{18-21}$ Prior to COVID-19, Crs had been correlated with ARDS as it affected the amount of aerated lung volume; however, the prognostic value of $\mathrm{Crs}$ on mortality remains unclear. ${ }^{22}$ Ultimately Crs was not included in the Berlin definition of ARDS secondary to the lack of evidence for its predictive validity. ${ }^{12}$ As the pandemic emerged, it was proposed that two phenotypes existed differentiating COVID-19 ARDS based on high vs low Crs. It was suggested this was atypical from non-COVID-19 ARDS. In reality, prior literature had not examined phenotypic variations of Crs in ARDS prior to the pandemic. This led Panwar et al. to perform a secondary analysis of the LUNG SAFE study to determine if Crs-base]d phenotypes exist in non-COVID-19 ARDS and whether Crs impacts outcomes. ${ }^{23}$ They found a wide range of Crs with one in eight patients having preserved Crs (Crs $>50 \mathrm{ml} /$ $\mathrm{cmH}_{2} \mathrm{O}$ ). Among those phenotype L patients, a significant portion had moderate to severe hypoxemia. Lower Crs on the first day of ARDS, was independently associated with higher mortality, however there was no clear transition point suggesting arbitrary thresholds for phenotype definitions..$^{23}$
Panwar's findings among non-COVID-19 ARDS patients are comparable to our findings in the COVID-19 ARDS population. We also found a wide range of $\mathrm{Crs}$ with about one in eight patients having preserved $\mathrm{Crs}>50 \mathrm{ml} / \mathrm{cmH}_{2} \mathrm{O}$. While we did see an increasing risk of mortality with decreasing $\mathrm{Crs}$, we did not find a statistically significant difference in mortality when comparing phenotype $\mathrm{H}$ vs $\mathrm{L}$ as defined by $\mathrm{Crs}<30 \mathrm{vs}>30 \mathrm{ml} / \mathrm{cmH}_{2} \mathrm{O}$. Likewise, we did not find any clear transition threshold to define Crs phenotypes based on a mortality relationship.

Moving beyond diagnostic phenotyping of COVID-19 ARDS patients, it has also been proposed by proponents of the Crs phenotype concept that phenotype L patients should be managed with alternative ventilator strategies than the traditional approach to non-COVID-19 ARDS. These strategies would include low PEEP and high tidal volume to compensate for increased dead space caused by hypoxic pulmonary vasoconstriction. ${ }^{15,24}$ Following this postulation, several studies have confirmed heterogeneity of lung morphology among COVID-19 ARDS patients and concluded patient specific care along with an evidence-based approach to traditional ARDS management should remain the mainstay of COVID-19 ventilator management. ${ }^{25,26}$

Based on our findings, we propose the observed differences in compliance are part of a continuum of illness that is patient specific 
and not exclusive to or definable by specific phenotypes. We would continue to advocate for traditional lung-protective ventilation strategies in the COVID-19 ARDS population modified if needed by individual bedside assessment. . $^{21,27,28}$

\section{Limitations}

While this is one of the largest case series to date analyzing Crs among mechanically ventilated COVID-19 ARDS patients, this is still a small single center series that was retrospective in nature. While our clinical practice pattern was adherence to lung protective ventilator strategies as recommended by the ARDS network, management was per the intensivist's discretion and therefore may have had some heterogeneity. The collection of ventilation variables was limited to the first three days of mechanical ventilation following the onset of respiratory failure; we therefore cannot speak to the effect of Crs on mortality beyond day three of mechanical ventilation.

\section{Conclusion}

A wide spectrum of Crs was observed among COVID-19 ARDS patients requiring invasive mechanical ventilation. We found only $13.5 \%$ of these patients had preserved $\mathrm{Crs}>50 \mathrm{~mL} / \mathrm{cmH}_{2} \mathrm{O}$, which is consistent with the percentage of non-COVID-19 ARDS patients that have preserved Crs. While we identified a trend towards increasing mortality as Crs decreased, there was not an identifiable threshold marking a significant difference in mortality based on phenotypic definitions. We therefore would not define COVID-19 ARDS patients by phenotypes $\mathrm{H}$ or $\mathrm{L}$ and would not stray from traditional ARDS ventilator management strategies.

\section{Declarations}

Funding: The authors did not receive support from any organization for the submitted work.

\section{Conflicts of interest/Competing interests}

There are no conflicts of interest to declare.

\section{Availability of data and material}

There is transparency of data to support our manuscript.

\section{Code availability}

There is custom code availability that supports our manuscript.

\section{Authors' contributions}

All authors made substantial contributions to conception, design, data collection and editing of the manuscript.

\section{References}

1. Huang C, Wang Y, Li X, et al. Clinical features of patients infected with 2019 novel coronavirus in Wuhan, China. Lancet. 2020; 395(10223):497506.

2. WHO Director-General's opening remarks at the media briefing on COVID-19: 11 March 2020.

3. https://www.who.int/emergencies/diseases/novel-coronavirus-2019 .

4. University JH. The center for systems science and engineering (CSSE). 2020 .

5. Auld SC, Caridi-Scheible M, Blum JM, et al. ICU and ventilator mortality among critically ill adults with coronavirus disease 2019. Crit Care Med. 2020;48(9):e799-e804.
6. Abate SM, Ahmed Ali S, Mantfardo B, et al. Rate of intensive care unit admission and outcomes among patients with coronavirus: A systematic review and Meta-analysis. PLoS One. 2020;15(7):e0235653.

7. Tian $\mathrm{R}, \mathrm{Wu} \mathrm{W}$, Wang $\mathrm{C}$, et al. Clinical characteristics and survival analysis in critical and non-critical patients with COVID-19 in Wuhan, China: a single-center retrospective case control study. Sci Rep. 2020;10(1):17524.

8. Wang Y, Zhou Y, Yang Z, et al. Clinical characteristics of patients with severe pneumonia caused by the SARS-CoV-2 in Wuhan, China. Respiration. 2020;99(8):649-657.

9. Yang X, Yu Y, Xu J, et al. Clinical course and outcomes of critically ill patients with SARS-CoV-2 pneumonia in Wuhan, China: a singlecentered, retrospective, observational study. Lancet Respir Med. 2020;8(5):475-481.

10. Choron RL, Butts CA, Bargoud C, et al. Surgeons in surge - the versatility of the acute care surgeon: outcomes of COVID-19 ICU patients in a community hospital where all ICU patients are managed by surgical intensivists. Trauma Surgery \& Acute Care Open. 2020; 5:e00557.

11. Amato MB, Meade MO, Slutsky AS, et al. Driving pressure and survival in the acute respiratory distress syndrome. N Engl J Med. 2015;372(8):747755 .

12. Gattinoni L, Pesenti A. The concept of "baby lung". Intensive Care Med. 2005;31(6):776-784.

13. ARDS Task Force, Ranieri VM, Rubenfeld GD, et al. Acute respiratory distress syndrome: the Berlin Definition. JAMA. 2012;307(23):25262533.

14. Li X, Ma X. Acute respiratory failure in COVID-19: is it "typical” ARDS? Crit Care. 2020;24(1):198.

15. Gattinoni L, Coppola S, Cressoni M, et al. COVID-19 does not lead to a "typical" acute respiratory distress syndrome. Am J Respir Crit Care Med. 2020;201(10):1299-1300.

16. Marini JJ, Gattinoni L. Management of COVID-19 respiratory distress JAMA. 2020;323(22):2329-2330.

17. Gattinoni L, Chiumello D, Caironi P, et al. COVID-19 pneumonia: different respiratory treatments for different phenotypes? Intensive Care Med. 2020;46(6):1099-1102.

18. Kellum JA, Lameire N, Aspelin P, et al. Kidney disease: improving global outcomes (KDIGO) acute kidney injury work group: KDIGO clinical practice guideline for acute kidney injury. Kidney Int Suppl. 2012;2(1):1138.

19. Chiumello D, Busana M, Coppola S, et al. Physiological and quantitative CT-scan characterization of COVID-19 and typical ARDS: a matched cohort study. Intensive Care Med. 2020.

20. Beloncle FM, Pavlovsky B, Desprez C, et al. Mercat A: Recruitability and effect of PEEP in SARS-Cov-2-associated acute respiratory distress syndrome. Ann Intensive Care. 2020;10(1):55.

21. Ferrando C, Suarez-Sipmann F, Mellado-Artigas R, et al. Clinical features, ventilatory management, and outcome of ARDS caused by COVID-19 are similar to other causes of ARDS. Intensive Care Med. 2020.

22. Haudebourg AF, Perier F, Tuffet S, et al. Respiratory Mechanics of COVID-19- versus Non-COVID-19-associated acute respiratory distress syndrome. Am J Respir Crit Care Med. 2020;202(2):287-290.

23. Nuckton TJ, Alonso JA, Kallet RH, et al. Pulmonary dead-space fraction as a risk factor for death in the acute respiratory distress syndrome. $N \mathrm{Engl}$ J Med. 2002;346(17):1281-1286.

24. Panwar R, Madotto F, Laffey JG, et al. Compliance phenotypes in early acute respiratory distress syndrome before the COVID-19 Pandemic. Am J Respir Crit Care Med. 2020;202(9):1244-1252.

25. Tsolaki V, Siempos I, Magira E, et al. PEEP levels in COVID-19 Pneumonia. Crit Care. 2020;24(303). 
26. Ziehr DR, Alladina J, Petri CR, et al. Respiratory pathophysiology of mechanically ventilated patients with COVID-19: A cohort study. Am J Respir Crit Care Med. 2020;201(12):1560-1564.

27. Bos LDJ, Paulus F, Vlaar APJ, et al. Subphenotyping acute respiratory distress syndrome in patients with COVID-19: Consequences for Ventilator Management. Ann Am Thorac Soc. 2020;17(9):1161-1163.
28. Botta M, Tsonas AM, Pillay J, et al. Ventilation management and clinical outcomes in invasively ventilated patients with COVID-19 (PRoVENTCOVID): a national, multicentre, observational cohort study. Lancet Respir Med. 2020.

29. Fan E, Beitler JR, Brochard L, et al. COVID-19-associated acute respiratory distress syndrome: is a different approach to management warranted? Lancet Respir Med. 2020;8(8):816-821. 